\title{
AIRBUS efforts towards advanced real-time Fault Diagnosis and Fault Tolerant Control
}

\author{
Philippe Goupil*, Josep Boada-Bauxell*, Andres Marcos***1 Emmanuel Cortet*, Murray Kerr***, Hugo Costa*** \\ *AIRBUS, Aircraft Control, 316, route de Bayonne, 31060 Toulouse Cedex 09, France \\ (+33-561183803; \{philippe.goupil\}\{josep.boada-bauxell\}\{emmanuel.cortet\}@airbus.com). \\ ** University of Bristol. Aerospace department - Queen's Building. University Walk. Bristol, BS8 1TR. UK \\ andres.marcos@bristol.ac.uk \\ *** DEIMOS Space S.L.U., Ronda de Poniente, 19, 28760, Tres Cantos, Madrid, Spain \\ \{murray.kerr\}\{hugo.costa\}@deimos-space.com
}

\begin{abstract}
In this article the industrial goals and objectives of the European Framework 7th project termed "REconfiguration of CONtrol in Flight for Integral Global Upset Recovery (RECONFIGURE)" are presented. Commercial aircraft fault tolerant control (FTC) strategies in the flight control system (FCS) are based on fail-safe flight control law reconfiguration which relies on upstream hardware redundancy-based robust Fault Detection and Diagnosis (FDD). This industrial state-of-practice fits well in the current certification process but it also decreases the easiness of the piloting task as soon as the system level of degradation increases. Thus, the main goal of RECONFIGURE is to research and develop aircraft FDD and FTC technologies that facilitate the automated handling of off-nominal/abnormal events and optimize the aircraft status and flight. The article details the project description of work, from industrial benchmark (fault scenarios and aircraft model) up to industrial verification and validation (V\&V) activities, via advanced FDD/FTC research and development. The expected results and perspectives are also presented.
\end{abstract}

Keywords: Aerospace Engineering, Flight Control, Fault Tolerant Control, Fault Detection and Diagnosis, Validation, European Project.

\section{INTRODUCTION}

In this article the industrial goals and objectives of the European Framework 7th project termed "REconfiguration of CONtrol in Flight for Integral Global Upset REcovery (RECONFIGURE)" are presented. The project aim is to support the investigation and development of advanced aircraft guidance and control (G\&C) technologies that facilitate the automated handling of off-nominal and abnormal events, while simultaneously alleviating the pilots' task and optimizing aircraft performance. This automatism and optimization must be performed while maintaining the aircraft current safety level which is compliant with presentday regulations. In addition, anticipating more stringent future regulations, this work could also contribute to advance the current safety level.

To provide the context for the proposal and timely relevance of the project, note that commercial aircraft FTC state-ofpractice in FCS as applied by aircraft manufacturers is based on fail-safe approaches. Within Airbus philosophy, in the event of an abnormal situation a nominal (so-called "normal") control law is switched first to a robust ("alternate") solution and then, if necessary, to a "direct" law [3]. This flight control law reconfiguration strategy relies on an upstream hardware redundancy-based robust FDD [3].
This industrial state-of-practice fits well in the current certification process. However, it also increases the workload of the handling task as the system level of degradation increases (see dashed line in Figure 1).

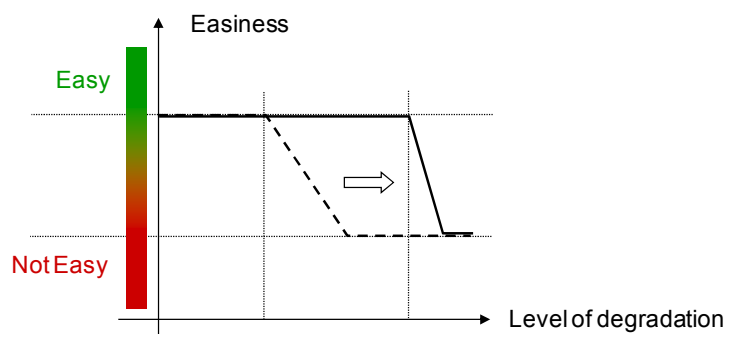

Figure 1: easiness of handling task in function of the system level of degradation

For the future aircraft, the need to change the design paradigm towards a performance-oriented one has been identified. We will term this new paradigm as: "Full-time \& all-event availability of performance-optimized G\&C functions". This paradigm can be translated into extending the availability of the G\&C functions designed to assist the pilot and making the flight task easier. This can be represented by the shift depicted in Figure 1 from the dashed (current) to the solid line (the wished innovation). Thus, the

\footnotetext{
${ }^{1}$ This author was with Deimos Space in charge of proposal and project coordination until the 1st project meeting. He gladly acknowledges that all his work in this project until that time was performed at Deimos Space.
} 
main goal of RECONFIGURE is to research and develop new fault diagnosis and reconfigurable control methods (FDD and FTC) possibly fed by Flight Parameter Estimation (FPE), with the ultimate goal to extend as long as possible the nominal $\mathrm{G} \& \mathrm{C}$ functionalities in order to simplify the handling of off-nominal/abnormal events and optimize the aircraft status and flight.

Starting with the motivation for the first technology highlighted before, FDD, even if rarely broached in the literature it is now recognized that advanced FDD can contribute to the future more sustainable aircraft [1][2]. Indeed, it can be demonstrated that improving FDD performance in FCS allows to enhance the aircraft structural design (resulting in weight saving), which in turn helps increase aircraft performance and optimize its environmental footprint (e.g. fuel consumption, noise). Thus, advanced FDD for early and robust detection of incipient faults is of primary interest for the development of the future cleaner, quieter, smarter, more affordable and easier to fly aircraft. With respect to the FPE technology, extending the G\&C functions could require to prolong the availability of some key flight parameters which might require novel estimation techniques. For example, "Virtual" sensors derived from mathematical models (analytical redundancy) could be an interesting solution, This is why FPE is also an important component of the project. With respect to the FTC technology, note that the main goal of using these techniques in flight control is to facilitate the aircraft control in case of anomalous or upset conditions (i.e. failures or changes of flight parameters) when the aircraft is still flyable given the available control power. Thus, the applicability of FTC techniques is strongly conditioned by the availability of an upstream reliable and prompt FDD system which could necessitate FPE. Therefore, the development of integrated FDD/FPE-based FTC techniques is one important goal of RECONFIGURE.

This paper is organized as follows: in section 2 the objectives and structure of the project are detailed from (i) the industrial benchmark and assessment tool, through (ii) research and development of advanced FDD/FTC, and up to (iii) industrial V\&V activities. Section 3 presents the Airbus benchmark scenario. Section 4 is devoted to the aircraft model that will serve as part of the benchmark. A panorama of the academic techniques considered by the consortium is presented in section 5. Finally, section 6 details the validation tools that will be used to assess the viability and performances of the proposed designs to tackle the Airbus benchmark.

\section{OBJECTIVES AND DESCRIPTION OF WORK}

RECONFIGURE is a three-year project that started in January 2013 and is co-funded by the European Community under the $7^{\text {th }}$ Framework Programme. The consortium is composed of 8 beneficiaries from 6 European countries and is coordinated by Deimos Space S.L.U. (Figure 2). It establishes a good balance of organizations' background (industry, research labs and Universities) with ample experience in the diverse pertinent fields: aerospace, FPE, FDD and FTC. The aforementioned project objectives are achieved by pursuing four main scientific and technological objectives:
1) Advanced parameter estimation and fault diagnosis approaches: it should be noted that most of the diagnosis methods that will be considered arise from the previous FP7 project ADDSAFE (2009-2012) [1]. This precursor project investigated FDD techniques for optimizing the structural design and aircraft performance in face of faults of aircraft sensor and actuator main components. RECONFIGURE can be considered in its entirety as the next step of ADDSAFE and particularly to this specific objective as a continuation, but with the notable change of focusing on abnormal events directly affecting the FCS.

2) Reconfigurable G\&C approaches: RECONFIGURE will consider techniques allowing adjusting, or even adapting, to abnormal in-flight events. This will make great strides towards a "full-time availability, all-event performanceenhancement FCS" since for each situation the control law will be adjusted, reconfigured and/or adapted to the best aircraft condition while enhancing the performance. The techniques to be developed will try to maintain the current G\&C mission optimal functionalities continuously operating, or at least for as long as possible, and when unavoidable, smoothly switch between the state-ofpractice "normal", "alternate" and "direct" control laws.

3) Integration issues and approaches for estimation, diagnosis and G\&C: although the two above objectives could be envisioned from an independent design perspective, in reality they are closely related on-board, especially if the FDD outputs feed the reconfigurable G\&C approach (active FTC). Thus, it is of primary interest to investigate the issues related to their integration from a practical perspective as well as to explore approaches that directly lead to integrated designs.

4) Clearance approaches for the above type of systems: the certification of the proposed FPE/FDD/FTC integrated or even individually technique is still rarely broached in the literature [4]. Current and past research projects have almost exclusively focused on the clearance of the stateof-practice flight control laws [5]. In the context of RECONFIGURE, the new challenge to be tackled is the clearance of the FCS as a single entity, that is, examination of the full adjustable / reconfigurable / adaptable FCS as a single time-varying component.

The last three objectives represent a significant step forward from ADDSAFE, whose demonstration of the maturity of the proposed model-based FDD techniques has opened up the possibility for proposing RECONFIGURE.

This EU project is divided in 3 main work-packages (WP). It strives to combine the synergies between the scientific and the technological partners at all levels of the project. Thus, most tasks include all partners. The WP breakdown follows a typical EU project architecture: from basic research levels, thru enabling technologies levels, up to integration level:

- WP1: Industrial Benchmark \& Assessment Tools. This is where mainly the aircraft model and the fault scenarios are defined including the V\&V process, tools and metrics.

- WP2: Advanced G\&C Design, Clearance Methods and Tools (main research and development activities).

- WP3: Industrial V\&V for evaluation of the technological readiness level (TRL) of the designs. It applies an industrial 
$\mathrm{V} \& \mathrm{~V}$ : (i) verification in a functional engineering simulator with Monte Carlo analysis complemented by worst-case search tools, (ii) validation in Airbus V\&V process, including pilot-in-the-loop tests using real avionics.

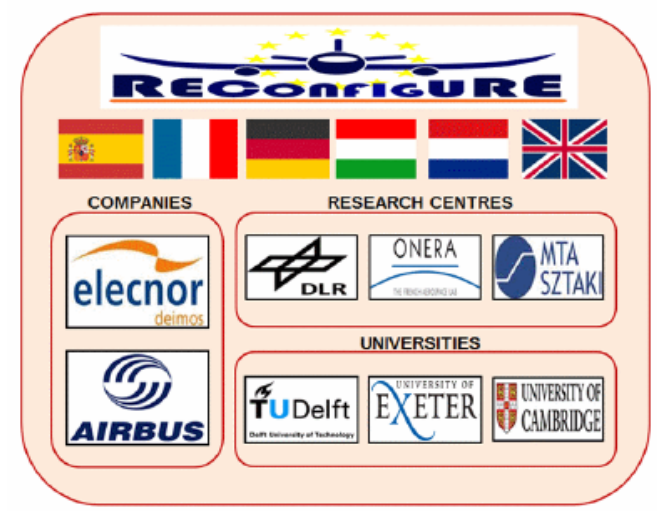

Figure 2: the RECONFIGURE consortium

\section{BENCHMARK SCENARIO DESCRIPTION}

The benchmark (WP1) includes the aircraft model development and the fault scenario definition. This section presents the three proposed scenarios, covering a wide range of events. The aircraft model is detailed in section 4 .

For industrial representativeness, the scenarios are not defined for a specific case (e.g. a single flight and aircraft configuration) but in useful domains of operation for design and validation purposes. Each domain is defined by a set of operating points (mass, center of gravity position, altitude and speed), pilot inputs and 3-D wind perturbations.

\subsection{Sensor faults}

\subsubsection{Detected loss of flight parameters (Sc1.1) -FTC \& FPE}

With electrical FCS for civil aircraft, the flight control law computation is fed by some key flight parameters [3]. The two underlying questions behind this first scenario are: (i) is it possible to develop flight control laws with a smallest set of flight parameters? And, (ii) what happens if some key parameters are lost during the flight (whatever the root cause)? The contemporary aircraft manufacturer state-ofpractice ensures a perfect coverage of this situation [3] but makes the piloting's task heavier. Extending G\&C and protection functionalities require an innovative approach.

Two key flight parameters have been chosen as a case study: the calibrated airspeed (Vcas) and the angle of attack (AoA). They are considered to be fully lost and this situation is detected and known by the pilots. Even if this configuration is known to be extremely improbable, it is not possible to theoretically demonstrate that it is impossible. There is no FDD requirement in this scenario. Inner-loop FTC requirements include maintaining the longitudinal normal law so as to be able to easily fly the aircraft manually. The outerloop objective is to maintain altitude hold and level change capability while keeping aircraft away from angle of attack and speed limits. Although this is a more FTC-oriented scenario, it is recognized that upstream FPE strategies could be also useful and complement the developments in here.

\subsubsection{Undetected erroneous flight parameters (Sc1.2) - FDD}

This second fault sub-scenario is closely connected to the previous in the sense that it corresponds to the situation preceding the detected loss of flight parameters. Due mainly to harsh electronic or environmental conditions, flight parameters measurements can have abnormal behaviours such as simultaneous additive or substitutive faults (e.g. oscillation, jamming, bias...). The same case study has been selected: Vcas and AoA flight parameters. Commercial aircraft are generally equipped with 3 dedicated sensors for each of these measurements. Thus, all possible fault configurations are envisaged, from 0 up to 6 simultaneous faults. The main FDD requirement consists in providing a valid and accurately voted (so-called "consolidated") flight parameter measurement for the control law computation and to isolate the faulty probes. The proposed design must be compliant with the following requirements: maximum acceptable error on the consolidated value (during static, dynamic, low and high speed phases), probability of false alarm (no degradation of the operational reliability) and of missed detection. FTC requirements are the same as for Sc1.1 with in addition the capability to keep efficient protections.

\subsection{Robustness to uncertain aerodynamic effects ( $S c 2)-F T C$ \& FPE}

This second scenario is dedicated to aircraft control and protection in icing conditions which can significantly modify the shape of the wings and thus the dynamics of the airliner. One of the main issues when dealing with ice on the wing is to demonstrate efficient protection by the FCS against stall. In this scenario, progressive ice accretion on the wing leads to a modification of the longitudinal aerodynamic coefficients degrading the closed loop response of the aircraft at high angle of attack. There is no FDD requirement. FTC requirements include an efficient AoA protection compliant with typical performance constraints. The design should be robust to different ice accretion shapes and to any other uncommanded wing surface motion (e.g. airbrakes runaway). FPE approaches could also be applicable here in support of the FTC activation (e.g. estimating aerodynamic coefficients changes). Finally, it should be noted that de-ice devices exist but their effect will not be taken into account in this work.

\subsection{Actuator faults}

3.3.1 Control performance and efficient flight envelop protection in case of detected actuator loss (Sc3.1) - FTC

It is mandatory to demonstrate efficiency (i.e. limited overshoot) of the flight envelope protection laws to maintain them active in case of detected actuator loss preventing the use of the concerned control surface. In this sub-scenario, a control surface is considered as fully lost after an abnormal event (e.g. faulty electronic component or mechanical breakage). The situation is known and the fault has been detected by a FDD strategy. There is no FDD requirement. FTC objective is to help maintain efficient manual control while keeping nominal AoA protection. Due to the reduction in the number of control surfaces, the aircraft response performance cannot be maintained for the full range of pilot 
inputs, thus the aircraft response should be maintained as long as the remaining actuators are not saturated (i.e. for small pilot inputs). In case of saturations, the aircraft response should be as fast as possible while respecting overshoot constraints.

\subsubsection{Detection and compensation of stall load (Sc3.2) - FDD}

A stall load situation corresponds to the occurrence of too heavy aerodynamic forces preventing the achievement of the commanded control surface deflection. The net sum of the antagonist forces reacted by the actuator are greater than the available hydraulic power. In these conditions, the control surface seems to be temporarily stuck at its current position. The robustness of some dedicated actuator or control surface monitoring can be impacted and the aircraft controllability can be reduced. The goal is to detect and confirm that the control surface faces a stall load phase and hence to discriminate with a faulty event and to estimate the achieved control surface deflection and the duration of the stall load phase. The proposed designs must be compliant with requirements on detection time and probabilities of false alarm and missed detection. FTC requirements as for Sc3.1.

\section{AIRBUS BENCHMARK}

The RECONFIGURE benchmark allows the partners to test their FDD/FPE/FTC techniques in fully realistic situations. To be fully representative of the aircraft and system dynamics, an in-flight validated nonlinear model (the yellow box in Figure 3 ) has been delivered, including all closed loop components (flight dynamics, actuators, sensors, flight control computers, etc). Because of the AIRBUS development framework, this model is presented as a black box with restricted input/output information. However, in order to enable the different partners to test their designs, part of the flight control computer is extracted into a Simulink model. This includes the baseline controller with interfaces to plug partners' designs. Finally, the benchmark architecture is completed by a Matlab-based interface that handles:

- The characteristics of the scenario to be simulated.

- The data flow from sensors outputs to the control laws.

- The commands computed by the control law to the aircraft model (actuators inputs).

- The synchronization of the incoming/outcoming data.

The fixed-step time behaviour of the simulation platform should be remarked. The communication between the control law commands and the model is performed at a fixed sampling period to ensure the data transfer. This characteristic limits the integration sampling time of Simulink which could become an important constraint to some FDD/FTC algorithms. However, considering the discrete behaviour of the actual flight control computers, the algorithms that cannot be run in this platform due to integration time limitation will not be able to be embedded in the actual aircraft hardware [3]. Therefore the platform provides a representative simulation mean to the partners to perform an initial validation of the FDD/FTC methods.

This benchmark is complemented with a simplified version of the aircraft model (Figure 4), which allows the partners to preliminarily tune their designs. The simplified model is composed of a linear model of the aircraft, the linear part of the baseline controller and simplified actuator/sensor models.

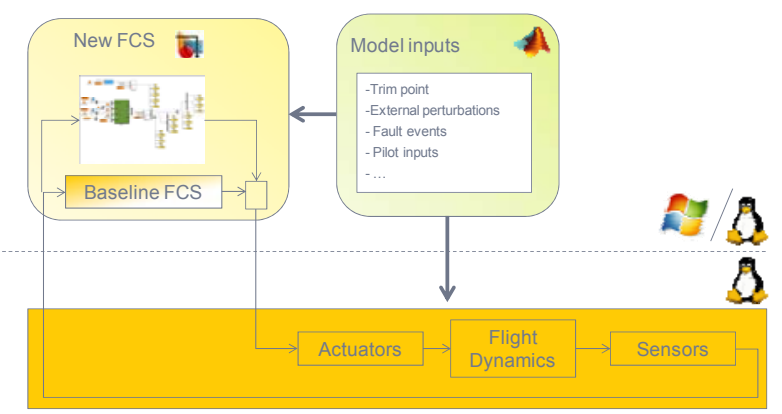

Figure 3 - Airbus benchmark architecture

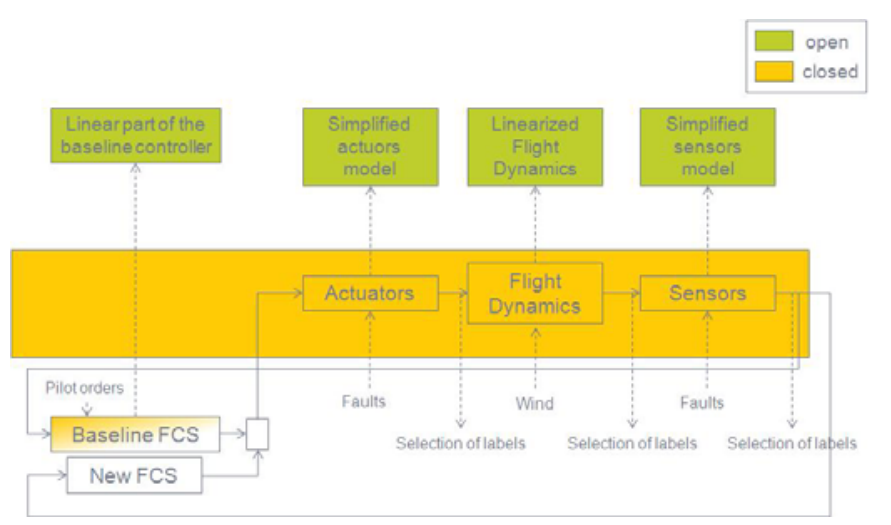

Figure 4 - Structure of the simplified benchmark

The linear model of the aircraft is a linearized version of the flight dynamics at a given flight point. However, the user has the option to define this flight point. Hence, the partners can choose as many design points as needed to tune the FDD/FTC algorithms. With regard to the linear part of the baseline controller, this is essentially the same law of the fully representative model without the compensations handling the time variant behavior of the aircraft. The simplified actuator model is a second order transfer function with rate and amplitude limitations while the sensor model is simplified to a filter and a time delay. Even if the models are only a simplified version of the fully representative aircraft, this benchmark still allow the user to define scenarios like described in Section 3, with a limit validity of the aircraft behavior at the surroundings of the linearized flight points. Finally, it has to be remarked that all the features of the simplified models are opened to the partners who can modified them if needed for FDD/FTC concerns.

\section{PANORAMA OF FDD/FTC METHODS}

The industrial FDD/FTC state of practice applied world-wide by all aircraft manufacturers relies on hardware redundancy and is fully compliant with current airworthiness regulations. Generalizing this approach to extend G\&C functionalities would result in unacceptable additional weight, complexity, cost and an increased overload on the pilots (Figure 1). This is why on the one hand, model-based analytical redundancy methods are of primary interest. Widely advocated by the academic community, most of these FDD/FTC advanced approaches rely on the idea of completed physical 
measurements with analytically computed redundant variables. On the other hand, data-based techniques are also interesting because the sensor data is directly exploited without explicit construction of a model. Several FDD/FTC techniques mastered by RECONFIGURE's partners cover both families: data-based and model-based approaches (Figure 5). A cursory presentation is made below to provide the reader with some non-exhaustive examples of the methods that will be investigated during the project.

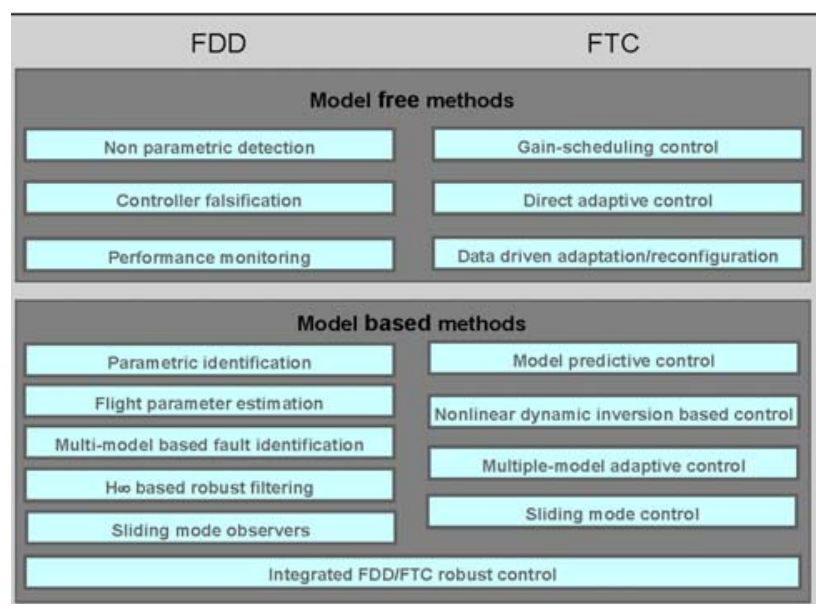

Figure 5: RECONFIGURE coverage of FDD and FTC methods

\subsection{FDD methods}

On-line parametric identification techniques to update a model or to compute a residual are appealing solutions [6]. FPE, as part of a FDD strategy, can be achieved via Kalman filtering, nonlinear signal processing or observers [7]. Especially adapted to very changing external conditions affecting the aircraft behavior, multi-model based fault identification could be well-suited [8]. Relying mainly on frequency domain design concepts, Hळ-optimization based methods will also be studied as they became increasingly important for FDD due to the explicit incorporation of robustness [9][10]. Sliding mode observers allows reconstructing un-measurable signals in the process being monitored by appropriate scaling and filtering of the socalled "equivalent output error injection". This makes a good candidate for fault estimation [11].

\subsection{FTC methods}

One class of FTC methods relies on explicit internal models of the aircraft. Typically, Model Predictive Control, which can be viewed as a particular indirect adaptive control method as it requires an on-line updated aircraft model, can be used for control reallocation after actuator failures, but also in the case of parametric faults (e.g. changes of aerodynamic coefficients) [12]. Fault accommodation could also be achieved thanks to adaptive control based on nonlinear dynamic inversion. The well-known robustness properties of sliding mode control with respect to so-called matched uncertainties could be investigated as well for fault tolerant control with respect to actuator or sensor faults [11]. Finally, an approach tackling the FDD/FTC interaction problem and the associated dual simultaneous design would be welcome, see for example [13].

The second class of FTC methods that certainly merits to be considered is the data-driven approaches (model-free). Already part of the industrial state-of-the-art, especially because of its limited computational effort, the gain scheduling technique can be coupled e.g. to LPV modeling to synthesize the scheduling parameters [14]. Direct adaptive control represents another opportunity, either simple or based on falsification techniques [15]. Finally, data-driven approaches leading to iterative controller improvement using batches of sample-to-sample data could lead to online FDD/FTC solutions [16].

\section{VALIDATION TOOLS}

A key step for the successful transfer to the aeronautics sector of the developed FDD/FTC methods is their demonstration on standardized industrial $\mathrm{V} \& \mathrm{~V}$ processes. $\mathrm{V} \& \mathrm{~V}$ facilities will be formed by a mix of desktop simulator (with full representative model) and industrial software assessment tools like the functional engineering simulator (FES) developed by Deimos. FES is a term used in Space to describe a software simulator describing at a functional level the components of a system and used in support of the specification, design and operations of space systems and for software V\&V activities [17]. This section gives an overview of the validation activities targeted in RECONFIGURE.

Considering the benchmarks provided, the validation of the proposed FDD/FTC methods will be performed according to the upward part of a conventional V-cycle, see Figure 6.

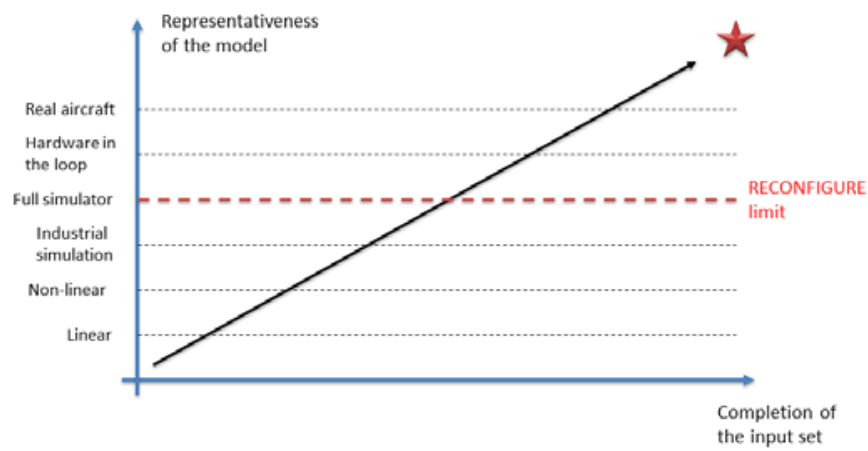

Figure 6 - Validation complexity evolution

Initially the partners can validate their design in the linearized model for several flight points. Once the tuning process is satisfactory the design should be applied to the non-linear and representative model of the aircraft and carry out a more exhaustive validation. Some guidelines are available for Guidance and Control law preliminary stage validation. They mainly consist in a list of operating points and maneuvers to be tested, which is obviously not exhaustive but that should provide a first quantitative feedback of the quality of the design. The tests to evaluate the designs' performance are organized in three steps in function of the validation set:

- Step1: evaluation of criteria on a list of given scenarios.

- Step2: statistical analysis of the criteria (random inputs).

- Step3: worst case criteria value. 
The initial validation process will be performed by using already existing industrial verification platforms [1] and/or new developed ones. The former is based on Monte Carlo campaign, the traditional clearance/certification approach. The main limitations of this approach are its large computational requirements (to obtain a sufficiently accurate solution and the coverage of the parameters. To overcome this limitation, an alternative approach will be used based on a continuous search of the parameter space to determine the worst-cases. This robustness analysis technique can be seen as an enhancement of the traditional gridding-based search and complementary to the Monte Carlo analysis. The main appeal of optimization-based clearance is its high reliability due to the continuous search using powerful global optimization techniques. DLR's proprietary optimization tool MOPS [18] will serve as the basis to develop a flexible clearance environment for FDD/FTC systems. The clearance requirements formulated in the previous task will be implemented as performance criteria to be minimized in order to achieve the worst performance corresponding to the worst-case parameter combination.

Hereafter, the compliant methods will be embedded in an industrial simulator with a representative cockpit. The goal is to allow Airbus test pilots to address the performance of the aircraft with and without the FDD/FTC algorithms in order to obtain a subjective but very important engineering judgment in the Airbus process design.

\section{CONCLUSION}

This article is an introductory presentation of the European RECONFIGURE project, covering its main motivations and objectives, describing shortly its work-package breakdown as well as the aircraft benchmark (fault scenarios and aircraft model). An attempt is made to summarize the FPE/FDD/FTC techniques considered within the consortium. Since one of the key objectives of the project is also the successful transfer to the aeronautics sector of the developed methods it is necessary to use $\mathrm{V} \& \mathrm{~V}$ processes and tools that are readily acceptable by industrial groups. This is the reason behind the use of a mix of industrial software assessment tools and simulators. The project strives to achieve an ambitious goal: the extension of G\&C functionalities to alleviate the pilots' task and optimizing aircraft performance towards a more easy-to-handle aircraft. Although the development of advanced FPE/FDD/FTC techniques can be considered nowadays as a mature field of research within the academic community, their application to real aircraft world has remained limited.

\section{ACKNOWLEDGMENTS}

This work was performed in the framework of the European RECONFIGURE Project: Grant agreement №: FP7-314544.

\section{REFERENCES}

[1] Goupil, P. and Marcos, A. Advanced Diagnosis for Sustainable Flight Guidance and Control: The European ADDSAFE Project. SAE AeroTech Congress and Exhibition, October 18-21, doi:10.4271/2011-01-2804.

[2] Zolghadri, A., Henry, D., Cieslak, J., Efimov, D., Goupil, P. Fault Diagnosis and Fault-Tolerant Control and
Guidance for Aerospace Vehicles - From Theory to Application. Series: Advances in Industrial Control. Springer. 2014, XVI, 216 p.

[3] Goupil, P. AIRBUS State of the Art and Practices on FDI and FTC in Flight Control System. Control Engineering Practice 19 (2011), pp. 524-539 DOI information: 10.1016/j.conengprac.2010.12.009

[4] $\mathrm{Hu}$, B. and Seiler, P. A Probabilistic Method for Certification of Analytically Redundant Systems. 2nd International Conference on Control and Fault-Tolerant Systems (SysTol'13), October 9-11, 2013 - Nice, France.

[5] Varga, A., Hansson, A. and Puyou, G. (Eds.) Optimization Based Clearance of Flight Control Laws, Lecture Notes in Control and Information Science, vol. 416, Springer-Verlag, Berlin, 2011.

[6] Hardier, G. and Bucharles, A. On-line Parameter Identification for In-flight Aircraft Monitoring. International Council of the Aeronautical Sciences Congress, Nice, September 2010.

[7] Van Eykeren, L., Chu, Q.P. and Mulder, J.A. Sensor Fault Detection using Adaptive Extended Kalman Filter. SAFEPROCESS 2012, Mexico City, Mexico, 2012.

[8] Varga, A. Least order fault and model detection using multi-models. Proc. of IEEE CDC09, Shanghai, 2009.

[9] Marcos, A., Ganguli, S. and Balas, G.J. An application of $\mathrm{H} \infty$ fault detection and isolation to a transport aircraft. Control Engineering Practice, 13:105, 2005.

[10] Kerr, M., Marcos, A., Peñín, L.F., Brieger, O., Postlthwaite, I. and Turner, M. Piloted Assessment of a Fault Diagnosis Algorithm on the ATTAS Aircraft. AIAA GNC 2009, Chicago, August 2009.

[11] Alwi, H., Edwards, C. and Tan, C.P. Fault Detection and Fault-tolerant Control Using Sliding Modes, AIC Series, Spring-Verlag, 2011.

[12] Maciejowski, J.M. Predictive Control with Constraints, Prentice-Hall, 2002

[13] Gaspar, P., Szabo, Z. and Bokor, J. An integrated vehicle control with actuator reconfiguration. 17th IFAC World Congress, Seoul, Korea, 2008.

[14] Lombaerts, T.J.J., Van Oort, E.R., Chu, Q.P., Mulder, J.A. and Joosten, D.A. On-line aerodynamic structure selection and parameter estimation for fault tolerant control. AIAA Atmospheric Flight Mechanics Conference, Chicago, USA, August 2009.

[15] Demourant, F., Ferreres, G. and Biannic, J.M. Falsification of an Aircraft Autopilot. Proceedings of the ACC, 2002.

[16] Dong, J., Kulcsar, B. and Verhaegen, M. Subspace based fault identification for LPV systems. SafePROCESS09, pp. 335-341, 2009.

[17] Fernandez, V., De Zaiacomo, G., Mafficini, A., Peñín, L. F., "The IXV GNC Functional Engineering Simulator," 11th Int. Workshop on Simulation \& EGSE facilities for Space Programmes, ESA-ESTEC, 2010

[18] Joos, H.-D. A methodology for multi-objective design assessment and flight control synthesis tuning. Aerospace Science and Technology, 3:161\{176\}, 1999. 